\title{
Les relations d'influence et leurs représentations
}

\section{Stéphane Laurens}

\section{OpenEdition}

\section{Journals}

Édition électronique

URL : http://journals.openedition.org/ress/2786

DOI : $10.4000 /$ ress. 2786

ISSN : 1663-4446

\section{Éditeur}

Librairie Droz

\section{Édition imprimée}

Date de publication : 27 novembre 2014

Pagination : $43-71$

ISBN : 978-2-600-01866-1

ISSN : 0048-8046

Référence électronique

Stéphane Laurens, "Les relations d'influence et leurs représentations », Revue européenne des sciences sociales [En ligne], 52-2 | 2014, mis en ligne le 01 janvier 2018, consulté le 19 avril 2019. URL : http:// journals.openedition.org/ress/2786 ; DOI : 10.4000/ress.2786 


\title{
LES RELATIONS D'INFLUENCE ET LEURS REPRÉSENTATIONS
}

\author{
STÉPHANE LAURENS \\ Université Rennes-2 - CRPCC \\ stephane.laurens@uhb.fr
}

\begin{abstract}
Résumé. Les recherches sur les techniques d'influence ou de manipulation alimentent le sens commun, étayent nos représentations et sont souvent utilisées pour donner sens à des événements qui sans cela seraient plus difficilement explicables (souvent des événements négatifs qui impliquent d'autres personnes que nousmêmes). L'analyse de 238 descriptions de situations d'influence (recueillies lors 18 entretiens semi-directifs) permet de décrire le lien entre les effets positifs versus négatifs attribués à l'influence et la nature de la relation d'influence. Nos résultats indiquent que l'influence est décrite comme ayant des effets négatifs lorsque la source est inconnue ou lointaine. À l'inverse dans les situations décrites comme vécues par nos informateurs, ou lorsque la source est proche, les effets de l'influence sont positifs. II semble que dans les représentations de nos informateurs, la possibilité d'établir une relation réciproque serait liée à des effets positifs de l'influence (ou négatifs en cas d'influence asymétrique).
\end{abstract}

Mots-clés: influence, manipulation, pouvoir, proximité, réciprocité, représentation, sens commun.

\begin{abstract}
Research on persuasion techniques or manipulation relies on common sense, supports our representations and is often used to decipher events that would otherwise be more difficult to explain (often negative events that involve other people but not ourselves). The analysis of 238 descriptions of influence situations (collected in 18 semi-structured interviews) allows us to describe the link between positive versus negative effects attributed to the influence and nature of an influence relationship. Results indicate that the influence is described as having a negative impact when the source is unknown or far away. In contrast, when influence is described as an experienced situation by persons interviewed, or when the source is near, the effects of influence are positive. It seems that it is the possibility of establishing a reciprocal relationship that leds to positive effects of influence (or negative in case of an asymmetrical form of influence).
\end{abstract}

Keywords: common sense, influence, manipulation, power, proximity, reciprocity, representation. 


\section{LES CONCEPTIONS DE L'INFLUENCE DIFFUSÉES PAR LA SCIENCE}

En psychologie sociale, l'influence a été assimilée au pouvoir (Moscovici, I979; Turner, 2005, p. 2), analysée sous l'angle du conformisme aux majorités (Crano, 2012) et les expériences qui ont fait la notoriété de la psychologie sociale sont précisément celles qui illustrent la soumission (Prislin, Crano, 20I2, p. 334). Dans les théories comme dans les expériences, l’influence apparaît comme un rapport asymétrique mettant en scène une source dotée de ressources psychologiques (crédibilité, autorité, pouvoir, prestige, attractivité, supériorité numérique, etc.), et une cible qui en serait dépourvue. Le symbole de ce récepteur passif est le sujet naïf des expériences (Laurens, Markova, 201 I).

À cette conception de la cible se sont ajoutées les études sur la manipulation, la persuasion, l'engagement, qui ont connu un fabuleux succès en psychologie sociale, mais aussi auprès d'un public plus large comme en témoigne la multitude d'ouvrages qui décrivent ces techniques, les présentant souvent comme des recettes faciles à mettre en ouvre afin de transformer le lecteur en manipulateur qui pourra alors obtenir ce qu'il désire (ou qu’il puisse se protéger des manipulations d'autrui) par le truchement d'une cible manipulée : on citera, notamment, Influence. Soyez celui qui persuade. Ne soyez pas celui qu'on manipule (Cialdini, 1987); Yes!: Devenez un as de la persuasion en 50 leçons (Goldstein, Martin et Cialdini, 2008) ; Le pouvoir de l'illusion: Les secrets de la persuasion, les clés de votre réussite (Paget, 2005) ; Petit traité de manipulation à l'usage des honnêtes gens (Joule et Beauvois, I987); Petit livre de Manipulation: 300 trucs et astuces pour obtenir tout ce que vous voulez (Azzopardi, 20I3); Manuel de manipulation pour obtenir (presque) tout ce que vous voulez (Conti, 2008), etc.'

I À côté de ces livres-recettes qui décrivent (principalement quand ce n'est exclusivement) les interactions concrètes entre individus, les concepts d'influence et de manipulations sont aussi mobilisés pour comprendre des phénomènes complexes comme l'émergence de nouvelles normes dans la mondialisation ou diverses décisions des pouvoirs publics. Par exemple dans La France un pays sous influence? (Éd. Vuibert, 20I2), Claude Revel analyse les processus d'influence des États, ONG, think-tank, lobbys... dans les prises de décisions, établissement de normes, de lois au niveau national et international. Autre exemple, dans les marronniers des hebdomadaires on trouve le pouvoir ou l'influence des francs-maçons, des juifs, des sectes, des lobbys... (voir pour les francs-maçons: <http://www.psychologie-sociale.com/index.php?option=com_ content\&task = view\&id $=\mid 64 \&$ ltemid $=76 \&$ limit $=\mid$ \&limitstart $=7>$, consulté le 8 mars 20 |4). 
Ces conceptions de l'influence et de la manipulation agrémentées de quelques idées colorées comme le lavage de cerveau, la programmation, la cascade d'engagement, etc., se sont largement répandues. En outre, elles ont trouvé audience auprès des institutions les plus importantes comme en témoigne cette affirmation tirée d'un rapport de l'Assemblée nationale (2000, p. 30) : «les instruments dont disposent les manipulateurs sont aujourd'hui sans commune mesure avec ceux des décennies passées ». Supposant l'existence de manipulations puissantes, l'Assemblée voulut alors protéger les individus par l'instauration d'un délit de manipulation mentale permettant d'interdire à des sources potentiellement néfastes d'exercer leurs mauvaises influences². Des réactions similaires apparurent à chaque fois que l'on crut en l'existence de techniques d'influence ou de manipulation puissantes. Ainsi, alors que l'hypnose était à la mode (utilisée pour réaliser des interventions chirurgicales, soigner diverses pathologies, montrée en spectacle, etc.) une demande d'interdiction de son utilisation fut votée, lors du Congrès international de l'hypnotisme expérimental et thérapeutique de I889 (Laurens, 2005). Certains pensaient qu'un hypnotisé pouvait être violé, volé ou même utilisé pour commettre un crime ${ }^{3}$. Aujourd'hui ces crimes sous hypnose n'effraient plus personne, mais ce sont les manipulations sectaires qui ont endossé ce rôle

2 La manipualtion consiste à exercer sur un individu « des pressions graves et réitérées ou à utiliser des techniques propres à altérer son jugement afin de la conduire, contre son gré ou non [...] à un acte ou à une abstention qui lui est gravement préjudiciable» (voir la section intitulée «De la manipulation mentale», et notamment l'article 225-16-4 du Rapport n²472 présenté à l'Assemblée nationale (2000). Cet article a finlament été rejeté, car notamment considéré comme liberticide.

3 Une enquête de Crocq (1896, p. 445) sur l'opinion de 58 spécialistes de l'hypnose, permet de savoir que pour $97 \%$ d'entre eux, le viol sous hypnose est possible, pour $88 \%$ le vol et le faux sont réalisables et $80 \%$ pensent même que le crime est possible.

4 Voir les rapports MIVILUDES (<http://www.miviludes.gouv.fr/publications-de-la-miviludes/ rapports-annuels $>$, consulté le 8 mars 20I4). 


\section{LES CHOSES SONT CE QUE LES GENS CROIENT QU'ELLES SONT}

Dans cette recherche, l'objectif n'est pas de savoir si des influences ou des manipulations puissantes existent réellement et peuvent produire tel ou tel effet, mais plus simplement de voir comment les gens se les représentent.

Cette question n'est pas secondaire car, comme on put le constater avec la possession, le magnétisme ou l'hypnose, par exemple, même si la technique utilisée ne produit aucun effet par elle-même, il suffit d'y croire pour trouver des individus se pensant sous influence, des individus utilisant ces techniques d'influence et d'autres individus luttant contre de supposés manipulateurs utilisant ces techniques. Des travaux clés ont affirmé cela à différentes époques :

- Malebranche (1946 [1674], t. II, p. 207-208), ayant analysé la possession, conclut qu'il ne fallait pas punir les sorciers, car en le faisant on renforçait la croyance en leur efficacité. Il convenait plutôt de se moquer d'eux et ainsi ils disparaîtraient comme ceux qui les pourchassent et ceux qui se prétendent victimes de leurs sorts ;

- le rapport des commissaires du roi de 1784 sur le magnétisme démontra que le magnétisme n’avait aucun effet, mais que l'imagination de ceux qui se croyaient magnétisés en avait et c'est par elle seule que les commissaires expliquèrent les faits attribués aux passes magnétiques ;

- Delbœuf (1993 [1893]) ou Bernheim (1911) réalisèrent des expériences de traitements par hypnose sans hypnotisation et ils obtinrent les mêmes effets que le patient soit hypnotisé ou qu'il croit l'être. Ils en arrivèrent même à la conclusion que l'hypnose, en tant qu'état nerveux spécifique, n’existe pas.

Ces effets des représentations et des croyances se manifestent dans les réactions liberticides qui visent à mettre sous contrôle le rapport à autrui (a-t-il le droit d'exercer de l'influence ?) et l'intimité du sujet (est-t-il responsable de son acte ou a-t-il été influencé ?). En effet, si le contrôle social peut être considéré comme un moyen pour faire face à des faits réels, il peut aussi être la réaction à une croyance en l'existence d'influences et de manipulations efficaces et néfastes qui, en réalité, n’existent pas. 
Ces représentations de l'influence se repèrent comme nous l'avons déjà vu dans la diffusion de conceptions scientifiques vers le grand public (grâce à des ouvrages, des reportages, des articles de presse, etc.) mais aussi dans les usages et les discours. $\mathrm{Si}$, dans ce texte, nous analyserons principalement les discours à l'aide d'entretiens semi-directifs, il convient préalablement de considérer les usages de ces notions d'influences et de manipulations.

\section{LES USAGES DE L'INFLUENCE Expliquer un événement négatif affectant autrui}

Comme l'indiquent les titres des ouvrages de vulgarisation de ces notions, on peut tout d'abord supposer que certaines personnes apprennent et utilisent des techniques pour influencer autrui, le manipuler. Cependant malgré la large diffusion de ces recueils de recettes et même de la place de ces techniques dans certaines formations, cet usage semble a priori limité à des populations très particulières : ceux qui travaillent dans la vente ou le management par exemple.

À côté de cet usage spécifique qui nécessite un apprentissage, il existe un autre usage bien plus fréquent et spontané qui consiste à expliquer des événements ou des conduites comme résultant d’influences. Or, comme nous allons le voir, la nature de ce qui est expliqué et la manière dont les choses sont expliquées par l'influence dépendent des représentations de ce qu'est une relation d'influence, en l'occurrence la relation asymétrique d'une source omnipotente face à une cible assujettie.

\section{I. L'AUTRE EST INFLUENCÉ}

La notion d'influence est mobilisée pour expliquer des décisions ou des comportements d'autrui. Par exemple, dans la Recherche de Proust, certains personnages se scandalisent du parti-pris dreyfusard de Robert (Saint-Loup) et, en bons Français, ainsi que le constatait Moscovici (1979, p. 28), ils en cherchent les raisons dans l'influence d'une femme:

C’est surtout comique, répondit la duchesse, étant donné les idées de sa mère qui nous rase avec la patrie française du matin au soir. 
- Oui, mais il n'y a pas que sa mère, il ne faut pas nous raconter de craques. Il y a une donzelle, une cascadeuse de la pire espèce, qui a plus d'influence sur lui et qui est précisément compatriote du sieur Dreyfus. Elle a passé à Robert son état d'esprit. [...] c'est probablement sa petite grue qui lui aura monté le bourrichon (Proust, 1946 [1922], p. II7-I22) .

On peut toujours supposer qu'un événement anormal ou surprenant est le résultat visible d'influences ou de manipulations invisibles. Ainsi, Alphonse Daudet décrit, dans son roman L’Évangéliste, la réaction de la mère d'une jeune femme majeure qui a décidé d'entrer dans une secte protestante et de rompre tous liens avec ses proches. Elle en informe sa mère par une brève lettre et cette dernière est complètement désemparée face à cette décision qui est, pour elle, incompréhensible. Ne pouvant croire au consentement de sa fille, elle croit qu’on lui a «dicté ces phrases monstrueuses dont elle ne pensait pas un mot» (Daudet, I888, p. 20I) et suppose alors l'existence d'influences, de manipulateurs (ibid., p. 208). Sa fille, si proche, avec qui elle vivait agréablement jusque-là ne peut pas avoir voulu cette rupture, c'est donc qu'on l'a manipulée. Avec cette explication par les influences ou les manipulations, tout rentre dans l'ordre: sa fille qu'elle aime et dont elle est assurée de l'amour ne peut être à l'origine de cette rupture et c'est un méchant manipulateur qui en est la cause.

L'influence ou la manipulation servent à expliquer de l'extérieur un acte du sujet. Ce n'est pas lui qui agit, mais il est agi par autrui ${ }^{6}$. Il ne consent pas véritablement puisque le consentement est potentiellement le résultat d'une manipulation exercée sur lui. Ainsi un acte peut être réalisé sous influence et en plus celui qui l’a réalisé peut croire qu’il l'a voulu alors qu’il était manipulé pour vouloir cet acte et le réaliser. Avec l'influence et la manipulation, il est inutile de demander à l'influencé s'il est influencé et la justice est donc «confrontée au consentement de victimes majeures et, apparemment, saines de corps et d'es-

5 Bloch ou Swann ne voient pas leurs positions dreyfusardes ainsi expliquées. Pour eux l'influence de la «race» et du milieu suffit à rendre compte de leurs positions. II est dès lors inutile de suspecter une quelconque manipulation.

6 L'énergumène est, par definition, celui qui est agi, qui subit passivement (Rousselle, 1990, p. 134). Cette passivité n'est cependant pas une apathie du corps, mais une absence du sujet dans ce que fait son corps. Les actes réalisés par son corps et les paroles formées par sa bouche ne sont pas les siens, il est agi par un autre, transformé en agent de sa volonté ou de son désir (ibid., p. 137). 
prit » (Assemblée nationale, 2000, p. 30). Il faut donc savoir, de l'extérieur (grâce à un juge par exemple) si la personne est véritablement responsable de ses actes, ou si au contraire elle a été manipulée. Il faut aussi éradiquer les influences néfastes (par exemple, en contrôlant l'usage de l'hypnose ou, plus récemment, en essayant d'établir un délit de manipulation mentale, voir Laurens, 2005).

\subsection{LES AUTRES SONT INFLUENCÉS, MAIS PAS MOI}

Comme l'indiquent les exemples tirés de ces romans, les discours sur l'influence ne se font pas à la première personne, mais à la troisième. La personne suppose qu'un individu a été influencé par autrui et explique ainsi sa conduite. Plus généralement, ce sont seulement les autres qui sont influencés, mais pas nous-mêmes. Ce phénomène appelé «third-person effect»a été observé par Walter Phillips Davison (1983) qui constatait que lors d'un sondage, les personnes interrogées répondaient affirmativement à la question «les gens sont-ils influencés par la télévision? » alors qu’en même temps ils répondaient négativement à la question «Êtes-vous influencé par la télévision?» (voir Missika, 1994).

Pour l'analyse des représentations, cet effet est intéressant : d'une part, il marque une distance entre la situation où l'évènement décrit, et celui qui le décrit qui n'est pas celui qui le vit ou l’a vécu. D’autre part, il marque une sorte de flou, d'indéfini tant sur la source de l'influence ou de la manipulation que sur le processus d'influence lui-même.

Ces lieux communs «on manipule les gens»ou «les médias nous influencent» ne disent rien des mécanismes de la prétendue manipulation et rien non plus de la source qui manipule : le mode indéfini du on ou du ils dispensent d'incarner, d'objectiver la source et permet de la laisser dans un flou: l'utilisation de ces formules et des explications par l'influence dispense de préciser le qui et le comment de cette supposée manipulation.

7 Le Monde a récemment publié les résultats d'un sondage indiquant que $51 \%$ des Français pensaient que «ce n'est pas le gouvernement qui gouverne» car «on ne sait pas en réalité qui tire les ficelles». Les manipulateurs étant par ordre décroissant d'importance: la finance internationale, les chaines de TV ou la presse, d'autres pays, les groupes comme les FrancsMaçons, certains groupes religieux. (Jonathan Parienté, Le Monde, 3 mai 2013: <http://www. lemonde.fr/societe/article/2013/05/03/la-moitie-des-francais-croient-aux-theories-du-complot_3170348_3224.html>, consulté le 8 mars 2014). 
Ainsi, on - qui est indéfini - influence - on ne sait comment - et tout cela semble suffisant puisque ce sont ces éléments structurants qui sont déclinés dans de nombreuses explications de sens commun.

\subsection{LES ÉVÉNEMENTS NÉGATIFS ET INCOMPRÉHENSIBLES}

Un autre aspect intéressant qui semble caractériser les situations pour lesquelles une explication par l'influence est mobilisée est la survenue d'un événement négatif difficilement compréhensible. Comme l'indiquent les exemples tirés de ces romans, c'est pour rendre compte de conduites étonnantes et choquantes (par ex. le dreyfusisme du fils de la comtesse de Marsantes ; l'entrée dans une secte, etc.) que l'influence est utilisée comme explication.

De ce point de vue, les théories du complot apparaissent comme des architectures très élaborées qui regroupent diverses explications basées sur des manipulations souterraines d'événements variés et négatifs. Ainsi un événement comme la chute des tours jumelles le i s septembre 200I serait le résultat souhaité de tel groupe qui voudrait envahir l'Irak afin de mettre la main sur le pétrole... On perçoit immédiatement la valeur heuristique de telles explications: le monde s'ordonne et des événements indépendants prennent place dans un ensemble.

Lévy-Bruhl distinguait les causes premières ou véritables des causes secondes, ces dernières n'étant «que des instruments ou tout au plus des causes occasionnelles au service de la cause véritable, qui est ailleurs » (Lévy-Bruhl, 1949, 3 février 1939). Pour cette mentalité primitive, il est vain de s'attarder sur les causes secondes qui sont contingentes et protéiformes et seule la cause première est importante. Par exemple qu'importe que des individus détournent des avions pour les lancer sur les tours? Cette cause seconde aux effets visibles n’a d'intérêt que dans l'objectif final: elle aurait très bien pu être remplacée par l'assassinat du président américain, par exemple. Par contre, la cause finale est constante: mettre la main sur le pétrole de l'Irak.

Dans cette recherche, nous nous centrerons principalement sur ces deux aspects que nous analyserons conjointement: aspect négatif versus positif des effets attribués à une influence et participation ou non de l'informateur à cette conduite (《je » versus «on » ou «ils »). 
Nous faisons l'hypothèse que la valence des effets de l'influence est liée à la proximité de la personne à la situation décrite. Les effets de l'influence devraient être plus négatifs lorsque la distance est grande (situation non vécue, source d'influence inconnue, situation ou source abstraite) que lorsque la distance est faible (situation vécue, concrète, source d'influence connue).

Outre ces premières observations sur les usages de la notion d'influence, deux arguments importants conduisent à cette hypothèse :

1) Comme nous l'avons précédemment montré, les techniques d'influence ont «mauvaise presse » et on se méfie de ceux qui les utilisent, mais en même temps, comme l'indiquent les titres des ouvrages de vulgarisation sur les techniques de persuasion et de manipulation, chacun de nous pourrait utiliser ces techniques et en tirer bénéfice. Si nous-mêmes n’utilisons pas ces techniques pour atteindre nos objectifs, nous pouvons cependant croire que d'autres n'auront pas de scrupule à le faire. Ainsi, sur cette dimension moralité/immoralité si souvent structurante de nos représentations de l'autre (Sanchez-Mazas, Licata, 2005, p. 349), nous voyons une opposition entre d'un côté ceux qui connaissent et utilisent des moyens et d'un autre côté nous-mêmes qui refusons de les employer: ils tireront un avantage de cet usage immoral et ceci peut-être à notre détriment (en ce qu'ils vont jouir de ce qu'ils obtiennent et que nous n'obtiendrons pas, dans la mesure où, en utilisant ces moyens, ils ont un avantage sur nous et qu'ils pourront même nous manipuler). Les ouvrages de vulgarisation et autres reportages accrocheurs mettant en exergue les effets contrastés de l'influence (négatifs pour la cible et positifs pour la source) ont sans doute contribué à rendre saillantes les dimensions opposées de désir/crainte, bénéfice/perte, envie/ renoncement, etc. activant une projection dans la source. Une projection qui partant de l'envie, de l'identification (jouir des bénéfices qu'elle peut tirer des manipulations) amène à la jalouser et la haïr. Cette dynamique psychique d'identification projective (Klein, 1946) consiste à inventer une altérité affublée des tendances ou désirs que le sujet ne peut assumer (manipuler autrui et l'aliéner à son propre désir) en sorte que «les objets ou les humains sur lesquels les gens projettent leur agressivité en viennent à être craints en tant 
que source belligérante» (Joffe, 2005, p. 108) ${ }^{8}$. Une dynamique psychique qui conduit à imaginer des méchants (dont les figures varient selon les époques : accapareurs, riches, CIA, francs-maçons, diable, etc.) dans une fausse conscience (Mannheim, 1929) que Joseph Gabel illustre avec son analyse de l'opinion publique anglaise face aux crimes de Jack l'Éventreur : compte tenu de l'horreur de ses crimes, ce dernier «ne pouvait être qu'un étranger [...] ou, faute de mieux, une personne appartenant aux milieux les plus déshérités » (Gabel, 1970, p. 82) alors même que pour Gabel ses crimes prouvaient qu'il s'agissait d'un «personnage très victorien » (ibid., p. 83).

2) Avec une source d'influence proche, connue, celui qui est influencé peut prendre position, répondre et ainsi exercer une influence en retour. De ce fait, s'il est cible de l'influence d'autrui, il est en même temps source d'influence vis-à-vis d'autrui. À l'inverse avec une source lointaine, inconnue, pour une situation qui n'est pas vécue par la personne, mais par autrui, la réciprocité est impossible et donc l'influence est asymétrique, posant de manière définitive la cible comme récepteur passif de l’influence d’autrui (voir Moscovici, 1979).

De l'inconnu au très bien connu - en passant par tous les degrés du flou, pluriel, particulier, connu - la relation d'influence change de nature.

Lorsqu'on influence, ou lorsque les journalistes, les médias, les politiques... influencent, il nous est tout à fait impossible d'exercer une influence réciproque9, alors que lorsque notre conjoint, un ami ou même tel journaliste particulier exercent une influence, il est possible de l'influencer en retour (Laurens, 20ı3). De la sorte, l'influence de on ou de ils est asymétrique et potentiellement aliénante puisque celui qui est influencé n'est qu’objet de l'influence et non sujet

8 L'analyse que fit Jodelet de la folie et des relations aux fous illustre cette dynamique de la mise en altérité dans le cas particulier d'une personne effectivement proche physiquement et quotidiennement mais qui, menaçant notre identité, est mise à distance pratiquement et symboliquement (Jodelet, 2005).

9 Vis-à-vis de l'influence d'un journaliste particulier ou d'un patron clairement identifié, nous pouvons réagir. Si notre action peut rarement être immédiate comme c'est le cas dans le dialogue avec un proche, nous pouvons écrire au journaliste ou porter plainte contre lui. La société a prévu des modalités pour que s'exercent des influences retour: tribunaux, syndicats, courrier des lecteurs, associations de consommateurs, etc. 
d'influence. «Sans réciprocité, il n’y a pas d'alter ego, puisque le monde de l'un enveloppe alors celui de l'autre et que l'un se sent aliéné au profit de l'autre.» (Merleau-Ponty, 1945, p.410).

Si ces conceptions sont largement partagées comme nous le pressentons, elles n'en restent pas moins paradoxales, qu'on les aborde sous l'angle de l'efficacité des influences aussi bien que sous l'angle de leur capacité explicative.

Même si on suppose (ce qui reste à démontrer) que des sources lointaines voudraient exercer des influences et en tirer profit, ces influences lointaines ne peuvent avoir qu'un effet très limité (Lazarsfeld et al. 1944; Katz, Lazarsfeld, 1955) si on compare cet effet à celui d'influences de proximité que nous recevons sans cesse. Il est donc bien étrange de redouter ces influences lointaines a priori inefficientes.

Comme nous l'avons vu, ces influences n'expliquent rien. Elles apparaissent plutôt comme des signifiants flottants destinés à combler le vide laissé entre des choses bien mieux identifiées : d'un côté des intentions et des intérêts supposés et de l'autre les faits. Nos représentations sociales nous fournissent cet instrument commode de la pensée qu'est l'influence, il est en effet de leur ressort de nous permettre «de concevoir des objets qui existent sans être perçus ou qui sont perçus sans exister» (Moscovici, 20I2, p. 28). Les individus peuvent «ainsi imaginer des causes ou des principes invisibles» (ibid.). Ces causes sont, dans cette étude, les influences et autres manipulations.

\section{MÉTHODE}

\section{I.MATÉRIEL}

I8 entretiens semi-directifs (d'environ Ihzo) auprès de 9 hommes et 8 femmes (enseignants, journaliste, informaticien, commercial, retraité, étudiants, policier, éditeur, employé d’administration, gestionnaire d'établissement de loisir, ouvrier mécanicien, chômeur) sur les relations d'influence ont été réalisés ${ }^{10}$. Ces entretiens étaient structurés en 3 parties principales. Une

10 Je remercie les étudiants de mon cours sur la technique de l'entretien qui ont participé à cette recherche, recrutant des informateurs, réalisant et retranscrivant des entretiens. 
première partie était simplement destinée à amener l'informateur à réfléchir aux différentes dimensions des relations d'influences. Pour ce faire les relances consistaient à recentrer sur l'objet. Dans une seconde partie - la principale puisqu'elle représente environ les $2 / 3$ de la durée des entretiens - l'informateur était invité à donner des exemples de situations d'influence et à les décrire le plus complètement possible. Dans la dernière partie, l'informateur était invité à revenir sur les descriptions des situations précédemment évoquées afin d'obtenir des informations complémentaires (par ex., les effets de l'influence sont-ils positif ou négatifs? la source a-t-elle conscience de l'influence qu'elle exerce? Une source d'influence peut-elle être invisible... ?). Dans cette partie étaient également induites des situations d'influence spécifiques si l’informateur n’en avait pas parlé spontanément (par ex. l'influence des sectes).

\subsection{MÉTHODE D'ANALYSE Analyses thématiques des situations d'influence décrites}

Dans l'ensemble du corpus, les situations de relation d'influences décrites par les informateurs ont été repérées ${ }^{11}$ puis codées.

Chaque situation ${ }^{12}$ est codée sur ces trois dimensions :

- Vécu/non vécu: la situation a-t-elle été vécue par l'informateur luimême ou non (selon la présence ou non des marqueurs «Je», «moi», etc.) ?

- Abstrait/connu: la situation fait-elle apparaître une source d'influence identifiable? On peut distinguer l'influence provenant d'une source connue (Sarkozy, tel collègue de travail, mon frère, TF1, etc.) de celle provenant d'une source abstraite (les médias, les journalistes, un homme

II On retient les situations décrivant une relation d'influence avec autrui, un groupe, une institution, mais on rejette l'influence de phénomènes ou d'objets (influence de lectures, de Fukushima, d'un chat, etc.). L'influence d'un livre ou d'un événement comme Fukushima n'est retenue que s'ils apparaissent explicitement comme des médiateurs d'autrui dans la relation d'influence (par ex. un auteur ou un éditeur qui publierait tel livre pour montrer ses convictions). On ne retient pas les analyses sur l'influence elle-même, in abstracto.

12 Dans le texte chaque situation est citée avec le code suivant: une lettre (de A à R) indiquant l'informateur suivit d'une numérotation des situations décrites par cet informteur: par ex., B8 signifie qu'il s'agit de la $8^{e}$ situation d'influence décrite par l'informateur B. L'absence de chiffre derrière la lettre signifie qu'il ne s'agit pas d'un verbatim codé comme une situation d'influence. 
politique, une famille, etc.). Pour trancher des cas intermédiaires, le critère suivant est retenu: est-il possible d'exercer une influence en retour sur la source? Si oui, il s'agit d'une source connue; si non, il s'agit d'une source abstraite.

- Domaines : les situations sont catégorisées en fonction du domaine dans lequel elles se déroulent (amis, famille, politique, média, éducation, travail, religion, secte, groupe).

Enfin, on note comment l'informateur, dans sa description de la situation, a évalué les effets de l'influence (évaluation positive ou négative, pas d'évaluation).

\section{RÉSULTATS}

\section{I. LES DEUX INFLUENCES: LA «BONNE»ET LA «MAUVAISE»}

Les premières phrases des informateurs opposent les «bonnes» et les «mauvaises » influences. Cet aspect évaluatif, détaché de tout exemple, de tout contexte, de toute référence à quelque mécanisme que ce soit, apparaît comme une première étape incontournable de leur réflexion.

Le plus souvent cette ambivalence se traduit par l'opposition d'influences positives versus négatives, mais elle apparaît aussi avec des oppositions bonne/mauvaise, saine/dangereuse, faste/néfaste, bénéfique/maléfique, etc.

Il y a deux grands types d'influences : les influences bénéfiques et les influences maléfiques, bonnes et néfastes. (M)

Mauvaise influence c'est les pressions, les chantages, les... voilà. Et bonne influence c'est le bon usage de la communication, l'argumentation. (F)

Moi, je vois, quand on me dit influence, je vois néfaste... Ça peut être une influence positive aussi. (I)

Les effets sont généralement évalués à partir des conséquences de l'influence sur la personne influencée : 
Après il peut y avoir plusieurs types d'influence par exemple. Il peut y avoir aussi bien des influences positives qui... je ne vais pas dire qui influencent positivement, mais... qui vont dans le bon sens... social ou... dans le bon sens. Et d'autres types d'influences qui pourraient être néfastes ou négatives... qui... comment dire ?... qui emmènent l'individu ou le groupe vers le bas... je ne sais pas comment dire ça... qui font régresser en gros. (Q)

Parfois c'est à partir de l'intention (par exemple altruiste ou égoïste) de celui qui exerce une influence qu'est évalué l'effet produit.

L’influence, elle peut être bonne et puis, des fois, mauvaises. Ça dépend des buts quoi. (A)

Il y a deux sortes d'influences : l'influence positive et l'influence négative. Donc l'influence positive, c'est-à-dire que si quelqu'un peut t'influencer ça peut t'aider dans la mesure où il va te pousser plus loin [...] manipuler les gens pour qu'ils fassent ce que nous voulons. Mais dans ce cas-là c'est négatif. (R)

Si cette dimension évaluative de l'influence apparaît constamment en début d'entretien, par la suite, elle diminue considérablement pour n’apparaître que très ponctuellement. Tout se passe comme si cette dimension évaluative initiait la réflexion, l'analyse, mais était ensuite mise au second plan lorsque les informateurs décrivent des situations d’influence.

\subsection{ANALYSE THÉMATIQUE}

Dans le corpus, on repère 238 descriptions de situations d'influence dont seulement 28 sont présentées comme ayant été vécues par l’informateur (I \% \%). Parmi ces 238 situations, II4 sont explicitement évaluées par l'informateur. Elles sont évaluées négativement dans $64 \%$ des cas. Ce sont ces II 4 situations décrites et explicitement évaluées que nous analyserons.

\subsection{Situations vécues ou non}

Le premier résultat important est que l'influence décrite par les informateurs dans les situations vécues est toujours positive (I3 cas parmi les informateurs C, F, H, J, L, M, O, P) alors que dans les situations non vécues (Io I cas), elle est jugée négativement dans $72 \%$ des cas $\left(\chi^{2}(\mathrm{I})=4,77, \mathrm{p}<.029\right)$. 
Les situations vécues décrivent l'influence de proches: amis, famille, parents, collègues, patrons.

Moi qui ai eu la chance d'être élevé dans une famille très soudée, très proche donc forcément, forcément les liens d'influence sont très... s'exercent constamment, d'abord, par les parents... (Ci6)

Une influence qui apparaît sous l'angle du conseil, de l'aide, de l'apprentissage :

J'ai un peu calqué ma façon de faire sur mes trois patrons, parce que je trouvais que dans le domaine où ils exerçaient, dans ce qu'ils faisaient, ils étaient bons donc il n'y avait pas de raison pour que moi je n'y arrive pas. (PI)

En tout cas moi c'est mon expérience personnelle, j'ai eu l'occasion de rencontrer des enseignants qui ouvrent des portes sur des sphères auxquelles on n'aurait pas envisagé avant. (L6)

C'est l'argumentation de mes proches, mes parents les premiers, qui m'ont fait prendre une autre décision pour m'inscrire dans une voie qui me correspondait mieux. (Fı)

L'influence, dans ces situations vécues, peut aussi prendre la forme d'un suivisme, une forme de l'influence qui d'ordinaire est connotée négativement, mais qui apparaît positivement dès lors que la situation est décrite comme vécue :

Je me suis laissée influencer par les médecins. Là, on voit bien la supériorité, parce que eux savaient, tandis que moi. [...] C'était vraiment la compétence face à l'incompétence, carrément! [...] Et pour moi, ça a été une influence positive. (O6)

Un copain ma dit «achètes le même ordinateur que moi et je te l'installerai. ». [...] Et on a acheté le même. J’ai été sous influence totale. J’y connaissais rien. Il a été utile, mais je n’ai pas regardé s'il y avait moins cher ailleurs, je me suis laissé complètement guider. Influence totale... j’y connaissais rien. [...] il marche toujours [...]. Libre arbitre zéro. Si, de décider de faire confiance! Le libre arbitre c'était de décider de lui faire confiance. (Mı) 
Notons enfin que, dans ces situations vécues, l'informateur est plus souvent en position d'influencé que d'influenceur. Trois informateurs seulement $(\mathrm{C}, \mathrm{N}, \mathrm{P})$ décrivent des situations où ils exercent une influence sur autrui. Chacun de ces informateurs décrit une situation d'influence dans le cadre de son travail (C qui conseille ses clients et prétend influencer leurs décisions ; $N$, enseignant, qui lors des réunions de parents d'élèves a toujours ses copies pour les montrer, prouver ce qu'il dit et ainsi «faire passer la pilule »; P, vendeur qui a appris qu'il faut éviter les discours techniques, mais donner des exemples simples pour obtenir la confiance). Notons une exception, $\mathrm{C}$, qui décrit à lui seul la moitié des situations vécues où l'informateur est en position d'influenceur. Pour cette personne, l'influence et la manipulation sont utilisées dans le domaine du travail, mais aussi avec des amis :

Je sors avec deux-trois amis, nous allons déjeuner [...] Mais j’ai tout simplement envie d'aller manger là donc du coup, je vais manipuler, influencer pour faire pencher la balance de mon côté et du coup, nous irons déjeuner là où je l’ai décidé. (C24)

Cette situation est une exception: la seule non seulement sur les II4 situations explicitement évaluées, mais même sur les 238 situations repérées, qui fait apparaître un usage de l'influence ou de la manipulation comme des instruments destinés à satisfaire ses propres désirs. Il s’agit donc là d’un usage très marginal des techniques d'influence et de manipulation.

\subsubsection{Source connue/abstraite}

Si l'influence des sources connues (33 cas) est évaluée négativement dans $42 \%$ des cas, celle des sources abstraites (8I cas) l'est plus fréquemment : $73 \%$ des cas $\left(\chi^{2}(\mathrm{I})=9,42, \mathrm{p}<.002\right)$.

Notons toutefois que dans les 33 cas de sources connues, I2 sont aussi des situations vécues dont on sait que l'influence est toujours évaluée positivement. Si on analyse uniquement les situations non vécues, l'influence des sources connues (2 I cas) est évaluée négativement dans $66 \%$ des cas et celle des sources abstraites (80 cas) dans $74 \%$ des cas. La différence entre ces deux catégories n'est plus significative (c2(I) $=0,42, \mathrm{~ns})$. 


\subsubsection{Les situations par domaines}

La valence des effets de l'influence est fortement liée aux domaines dans lequel la situation d'influence décrite se déroule.

Sur la base de cette dimension évaluative, 3 types de domaines se distinguent : effets d'influences positifs, négatifs et mixtes. Dans les domaines des influences de proximité (amis, famille, éducation), les effets sont positifs. En revanche, dans les domaines où elle émane de sources lointaines ou inconnues (secte, média, politique, religion), les effets sont généralement décrits comme étant négatifs.

Tableau I. Répartition des I I4 situations d'influence

\begin{tabular}{|c|c|c|c|c|c|c|c|c|c|}
\hline & \multicolumn{2}{|c|}{ VÉCU } & \multicolumn{2}{|c|}{ NON VÉCU } & \multirow{2}{*}{ TOTAL } & \multicolumn{2}{|c|}{ VÉCU } & \multicolumn{2}{|c|}{ NON VÉCU } \\
\hline & POSITIF & NÉGATIF & POSITIF & NÉGATIF & & POSITIF & NÉGATIF & POSITIF & NÉGATIF \\
\hline AMIS & 3 & 0 & 5 & 0 & 8 & $38 \%$ & $0 \%$ & $63 \%$ & $0 \%$ \\
\hline ÉDUCATION & I & 0 & 5 & I & 7 & $14 \%$ & $0 \%$ & $71 \%$ & $14 \%$ \\
\hline FAMILLE & 2 & 0 & 4 & I & 7 & $29 \%$ & $0 \%$ & $57 \%$ & $14 \%$ \\
\hline GROUPE & 2 & 0 & 4 & 4 & 10 & $20 \%$ & $0 \%$ & $40 \%$ & $40 \%$ \\
\hline TRAVAIL & 5 & 0 & 4 & 6 & 15 & $33 \%$ & $0 \%$ & $27 \%$ & $40 \%$ \\
\hline RELIGION & 0 & 0 & 2 & 5 & 7 & $0 \%$ & $0 \%$ & $29 \%$ & $71 \%$ \\
\hline POLITIQUE & 0 & 0 & 4 & 25 & 29 & $0 \%$ & $0 \%$ & $14 \%$ & $86 \%$ \\
\hline MÉDIA & 0 & 0 & 0 & 14 & 14 & $0 \%$ & $0 \%$ & $0 \%$ & $100 \%$ \\
\hline SECTE & 0 & 0 & 0 & 17 & 17 & $0 \%$ & $0 \%$ & $0 \%$ & $100 \%$ \\
\hline TOTAL & 13 & 0 & 28 & 73 & 114 & $11 \%$ & $0 \%$ & $25 \%$ & $64 \%$ \\
\hline
\end{tabular}

Nota. Situations d'influence décrites en fonction des effets (positif/négatif) dans le domaine dans lequel elles se déroulent et de leur présentation comme étant vécue ou non (pourcentages de cette répartition indiquée en italiques).

\section{- Influences négatives :}

L'influence est toujours vue négativement dans les domaines des sectes (17 cas), des médias ( 44 cas) et très souvent négativement dans les domaines de la politique (29 cas, $86 \%$ de négatif) et de la religion (7 cas, 7I \% de négatif). Dans ces domaines, les situations ne sont jamais vécues. 
Dans le domaine des sectes, ces situations non vécues, sont en outre éloignées: certains informateurs précisent qu'ils n'y connaissent rien: j'en sais rien ( $\mathrm{FII}_{\mathrm{I}}$ ) ; j'imagine que ( $\mathrm{C}_{14}$ ); d'après ce que j'en ai compris (B2o) et l'un d'eux explique même que ce qu'il sait lui vient des médias :

Les sectes, on en entend beaucoup parler avec Tom Cruise [...]. J'avais vu des reportages sur ça, [...] où les, les, je sais plus comment on appelle les patrons des sectes, les grands patrons on va dire, qui sont très très forts pour influencer, euh, qui ont des techniques très recherchées, euh, ils donnent des techniques, pas des techniques, mais euh, mais c'est vrai que c'est presque scientifique leur truc, euh, assez facilement ils arrivent à sortir euh, ils ont besoin d'une minute pour convaincre [...]. Si vraiment, euh, si vraiment ça marche. (I)

L’influence des sectes est décrite comme étant tellement puissante, que pour certains informateurs ce n'est plus de l'influence, mais un au-delà de l'influence :

C'est plutôt du lavage de cerveau que de l'influence [...] C'est pas de l'influence, mais de la manipulation... (D9)

C'est vraiment du mauvais... [...] c'est même plus que de l'influence, là c'est complètement du lavage de cerveau, une manipulation! $\left(\mathrm{O}_{7}\right)$

La secte pour moi c'est vraiment un exemple de manipulation et d'influence sous une de ses formes paroxystiques. (BI9)

Dans une secte on lobotomise complètement les personnes pour les faire adhérer entièrement aux principes de la secte. (R8)

Pourtant cette super influence des sectes n'est évoquée qu'à la fin des entretiens et parfois il faut même relancer explicitement 9 informateurs (B, D, F, G, H, J, L, N, O) pour qu'ils abordent ce domaine. Voici, par exemple, la réaction d'un informateur à la suite d'une relance introduisant la notion de sectes après plus d'une heure d'entretien :

J’avais pensé à la religion moi. Et j'étais dans la religion non sectaire. J'y avais pensé un petit peu à un moment donné. J’avais pas pensé sous le terme des sectes mais euh... (N) 
À l'inverse, le domaine des médias vient spontanément et rapidement à l'esprit des informateurs. Pour eux, les médias nous conditionnent (BI5), nous noient $\left(\mathrm{G}_{4}\right)$, mentent $\left(\mathrm{K}_{3}, \mathrm{M} 7\right)$, font du bourrage de crane (O8, Pi3) manipulent l'information suivant leur intérêt (E8) sont cyniques, veulent de l'audimat, se moquant des conséquences de ce qu'ils montrent (Ji2) afin de placer les publicités et faire acheter (K3) et de gagner de l’argent (Ji2).

Le but c'est vraiment de vouloir créer de l'audimat, peu importe les moyens, on veut créer de l'audimat et donc on se sert de personnages. On leur demande d'avoir une personnalité qui n'est pas la leur et vraiment de la pousser à l'extrême pour créer de l'audimat, attirer les gens et ils se moquent un peu des conséquences que cela peut avoir sur les gens. Si, un gamin de 8 ans regarde Secret Story et voit qu'il y en a un il a un melon sur la tête, il va reproduire exactement la même chose, mais eux tout ce qui les intéresse c'est de gagner de l'argent. (Ji2)

Ici contrairement aux sectes, les médias sont connus de nos informateurs qui parfois mentionnent précisément une émission (Ji2), un journal (Eıo) une chaîne de télévision (B25). Les informateurs ont une pratique des médias et quelques-uns analysent leurs méthodes (la une de journaux [Eı]; la présence des médias [Bı6]; la répétition dans l'information $\left[\mathrm{B}_{15}, \mathrm{G}_{4}\right]$; la publicité [K3, PI4], etc.) et un informateur tente même de trouver des manières de réagir à leur influence (E8).

L’influence des médias est notamment objectivée par la publicité qu'ils diffusent et dont ils tirent leurs revenus. Une publicité qui semble efficace puisque, pour nos informateurs, elle peut créer des demandes (Gio), susciter des besoins $\left(\mathrm{H}_{4}\right)$ et pousser à acheter (F8). Ainsi un informateur donne un exemple précis de publicité pour un shampoing (M5) et un autre décrypte la publicité pour une lessive :

Dans la pub t'as pas le choix d'être influencé, c'est étudié pour, c'est le métier des gens qui planchent sur les sujets pendant des heures et des heures, ça coûte très cher c'est pas pour rien. ( $\left.\mathrm{P}_{14}\right)$

Le domaine de la politique est fortement lié à celui des médias puisqu'en même temps que les informateurs parlent de l'influence en politique, ils mentionnent les médias comme intermédiaires et affirment l'interdépendance de ces domaines, parfois même de manière très explicite à travers un person- 
nage comme Berlusconi et les médias italiens (E5) ou comme TFI considérée comme une chaîne aux mains du pouvoir. (B2)

Si la majorité des situations d’influence en politique est aussi négative que celle des médias, on trouve quatre exemples d'influence positive dans ce domaine.

Trois sont des situations d'influence minoritaires (Gandhi [G9], Kadhafi [Kı6], idées minoritaires [Cıo]) face à des influences majoritaires. Des influences minoritaires qui sont évaluées positivement même lorsqu'elles ont pour source une personne à l'image aussi négative que Kadhafi! L’Analyse des influences attribuées à Kadhafi permet de bien mesurer l'importance de la réciprocité : Kadhafi en tant que dirigeant en place est même comparé à Hitler ( $\mathrm{L} 7)$, il a une influence descendante qui produit de la soumission, du suivisme et qui est très négativement évaluée. Néanmoins Kadhafi avant d’avoir le pouvoir fut, selon un informateur, confronté au pouvoir du colonisateur, il résista donc à l'emprise du pouvoir en place et, par ses actions et prises de position, il eut, selon cet informateur, une influence libératrice (précisons que Kadhafi n’avait qu'un an lors de la décolonisation). Aussi c'est en opposition à l'influence du pouvoir en place que l'influence de Kadhafi est considérée comme positive.

Kadhafi, c'était ça au début. Les Italiens occupaient la Libye et lui il a combattu contre les Italiens auprès du peuple libyen. Du coup il a été vu comme celui qui sauvé la Libye de la domination italienne. Il était vraiment très aimé, c'était le héros en Lybie et du coup il s'est forgé une popularité. (Kı6)

Cependant, cette influence jugée positive se transforme une fois qu'il est au pouvoir. Il devient un chef narcissique, un tyran qui exerce une influence à sens unique puisqu'il ne reçoit pas les influences d'autrui :

Au début il devait sûrement prendre en compte l'avis des gens, du peuple, mais au bout d'un moment il n'a plus pris en compte ce que les gens voulaient. Il commençait à ne penser qu'à lui, il est même devenu limite un peu fou quelque part, la preuve il disait à la télé «le peuple libyen máaime» alors que ce n'était pas vrai. Il était même peut être aveugle, peut être il croyait que c'était comme à ses débuts, que les gens l'aimaient alors que ce n'était plus le cas du tout. Après il a instauré ses règles, il a tout maîtrisé dans le pays. (K) 
Le dernier cas particulier dans le domaine de la politique est celui de l'influence positive que la femme de François Mitterrand aurait eue sur ce dernier, contribuant à faire de lui un grand président. Dans ce cas (C28), les protagonistes de la situation d'influence sont connus et l'influence qui s'exerce entre eux est une influence de proximité.

Dans le domaine de la religion, les effets de l'influence sont négatifs dès lors que la religion est au pouvoir : la religion au pouvoir apparaît comme une dictature (Li8, KıI), elle soumettrait les individus (C29), leur fermerait l'esprit (I8).

On observe néanmoins deux cas d'influence positive dans ce domaine. Pour un informateur, la religion rassurerait et permettrait de lier les gens entre eux (EI2). Pour un autre, la religion en tant que source d'influence serait marquée par des intentions positives :

dans la religion catholique, on va dire «Je vais t'aider, je suis là pour te soutenir», on va dire la vérité, c'est vraiment là pour les aider et les soutenir. (J9)

Pour cet informateur, c'est cette intention de la source qui distingue religion et secte. Pour lui, les sectes mentiraient et abuseraient de la fragilité des individus : dans une secte, on va faire croire à l'individu qui rentre dans la secte «on va t'aider, on est là pour te soutenir » alors que finalement c'est juste pour arriver à lui retirer quelque chose, donc la du coup, oui, c'est vraiment une influence directe et négative. (Jıo)

Cet exemple illustre bien un déterminant de la valence de l'influence : pour cet informateur, la source religieuse croit à son message tandis que la source sectaire fait croire quelque chose à sa cible. Aussi dans le premier cas, même si la croyance est infondée, fausse... l'influence n'est pas négative : le source croit et c'est parce qu'elle croit qu'elle est prosélytisme afin de partager ses convictions avec autrui. À l'inverse, faire du prosélytisme sans croire dévoile le machiavélisme des sectes.

\section{- Influences positives :}

Les effets de l'influence sont toujours décrits positivement entre amis, et très souvent positivement dans la famille $(87,5 \%)$ et l'éducation $(83,3 \%)$. 
Selon nos informateurs, certaines influences positives conduisent à la socialisation à l'intériorisation des normes de groupe.

dans un cercle d'amis, il peut y avoir des tas de manières d'influencer sur les vies, sur les choix de vie, sur la conformité d'une forme de vie. Tiens, il y en a deux qui commencent à avoir des bébés donc tout le monde s'y met, machin achète une baraque puis il se fait une grande terrasse. Il peut y avoir influence et mimétisme aussi. Oui ça se rapproche peut-être et du coup dans influence et mimétisme je vois plus quelque chose où les pensées sont assez jointes et à un moment il y en a une qui se détache puis elle est rejointe par les autres. Du coup dans un cercle d'amis c'est plutôt... si on regarde globalement et si on imagine une forme d'équité, d'égalité dans les rapports, je ne parle pas de rapport de pouvoir là [...] un cercle d'amis où les relations sont horizontales et bienveillantes. (E6)

y a la bonne influence, de la personne avec qui, je sais pas, adolescent tu peux traîner. Et je ne sais pas, l'ami avec qui tu traînes qui est très sportif va t'influencer à faire du sport plutôt que d'aller te droguer. (FI)

un enfant [...] c'est un buvard, c'est assez facile de savoir ce qu'il se passe et ce qu'il se dit autour de lui. C'est une forme d'influence et en tant que parents je crois qu'on en use beaucoup, mais de manière bienfaisante systématiquement évidemment. (E3)

Elles viennent de personnes ayant un statut supérieur, reconnu comme compétent, expérimenté et s'appliquent sur des personnes moins compétentes ou expérimentées permettant de les former.

Dans l'enseignement [...] le but c'est qu'ils prennent la direction qui nous semble bonne parce qu'on sait qu'au final, elle porte ses fruits. Enfin je le vois plutôt comme ça. Donc euh, on ne manipule pas parce que la valeur derrière elle est noble, c'est-à-dire faire progresser l'élève. (Ni3)

Enfin, dans ces domaines, les informateurs soulignent régulièrement les intentions positives de ceux qui exercent consciemment une influence: ils veulent aider, conseiller, transmettre leurs valeurs.

Dans un groupe d'amis, il y a un des membres qui a un problème assez grave et bien tout le monde va être là pour en parler avec lui, pour le soutenir, pour l'aider et faire que ça aille mieux. Ça, pour moi c'est de l'influence qui est positive. On aide l'autre, on s'entraide entre nous pour arriver à surmonter une période difficile. Ça s’appelle de l’amitié pour dire les choses clairement. (J5) 
L'influence familiale et amicale, c'est-à-dire les amis qui nous soutiennent et qui sont là pour nous aider au moment où on est en train de sombrer ou de chuter, eh bien cette influence [...] est positive parce que c'est un soutien. (B2 I)

Les deux seuls cas d’influences négatives mentionnées dans ces domaines (amis, famille, éducation) sont :

- la possibilité que l'influence des parents soit forte au point d'empêcher les enfants de «réfléchir par eux-mêmes» (K7);

- l'identification pathologique d'une jeune femme à une enseignante (l'informateur la décrit comme imitant son enseignante au point de perdre sa propre personnalité).

Dans ces deux cas, il n'y a cependant aucune intention néfaste attribuée à la source. En effet, dans le premier cas, l'informateur précise «je pense que les parents veulent le maximum pour leur enfant, ce qu'il y a de mieux » $\left(\mathrm{K}_{7}\right)$. Dans le second cas, l'enseignante est imitée, mais c'est à la cible que l'informateur attribue les raisons de l'influence. Une cible qui est décrite comme une jeune fille fragile qui s'identifie à un modèle (I7). Quoique cette identification le soit à une enseignante, raison pour laquelle ce cas est classé dans la catégorie «éducation», elle relève d'une situation d'influence similaire à de nombreuses identifications à des stars qui, elles, ont été classées dans la catégorie «média».

\section{- Influences mixtes :}

Enfin, deux domaines occupent une position intermédiaire quant à la répartition des influences positives et négatives : le travail et les groupes.

Le cas du travail est intéressant puisque toutes les situations de travail vécues sont positives tandis que celles non vécues le sont seulement 4 fois sur ı. Les situations d'influence positives sont celles d'entraide entre collègues, d’apprentissages venant de collègues expérimentés vers ceux qui le sont moins, de managers qui peuvent motiver le personnel. Dans les situations d'influence positive non vécues de la catégorie travail, on retrouve les mêmes situations avec deux cas singuliers : celui de l'influence d'un syndicat sur un patron (D7) et celui de la résistance de salariés envers leur hiérarchie (A5) (à nouveau une forme d'influence minoritaire en opposition à une influence majoritaire). 
Tu as l'influence hiérarchique professionnelle, ça c'est l'état naturel on va dire... Après, en cas de conflit, tu as l'influence inversée... celle des syndicats: ils essayent d'influencer la direction ou le PDG [...] agissent davantage pour l'intérêt commun. (D7)

J'ai connu un service d'environ quarante personnes [... . Quelques personnes responsables de ce service sont convoquées pour qu’on leur donne les nouvelles attributions du service. Et puis ils reviennent dans leur service, font un compte rendu de la réunion avec la direction. Et rien n’a été décidé en faveur du service, des employés. Et donc, il y en a un qui se lève et qui dit mais, vous êtes des chefs, vous faites quoi, vous êtes qui, vous êtes nuls quoi... pourquoi vous vous êtes laissés menés comme ça... Et ce personnage, à tenir ce discours, un peu cru quand même, a eu une forte aura sur le service et aussi en raison de sa compétence technique. Et une semaine plus tard, le service était dans un autre état grâce à lui. Juste par cette réflexion. ( $\mathrm{A}_{5}$ )

Les situations d’influences négatives évoquées (aucune n’est vécue par les informateurs) sont présentées comme produisant de la soumission, du suivisme et même peuvent briser l'individu. Ces influences néfastes proviennent de supérieurs (manager, patron) qui exercent des pressions (par ex. Orange [M9]) sur des individus de statuts inférieurs (Li7). L’influence est décrite comme intentionnelle, décidée par la source à qui incombe donc les effets néfastes produits. Par exemple, un informateur parle de harcèlement au travail (B3) et d'un patron pervers qui trouverait une jouissance dans la manipulation des ses employés (B7).

Pour le groupe (dans cette catégorie on trouve les groupes d'insertion des individus avec des personnalités particulières comme les «fortes têtes», les «leaders», l'entourage - autre que la famille, les amis, etc. -, mais aussi la masse, le groupe), si la situation vécue est positive, les situations non vécues ne le sont qu'une fois sur deux.

L'influence a des effets positifs dans les cas du soutien d'amis (J5), le conseil d’un médecin (O6), le rôle positif du leader pour maintenir le groupe (J8), des personnes prestigieuses prises pour modèle par un enfant (Giz) et les groupes en tant qu'ils sont des relais de la famille dans la socialisation (L5).

Les effets négatifs apparaissent dans le cas de l'influence d'une forte tête qui peut amener un enfant à faire de sottises, à adhérer à des normes de groupes néfastes (L2), à cause de pressions au conformisme (C30, J4). Des mauvaises influences de l'entourage qui peuvent conduire à des conduites criminelles (Jiz). 


\section{DISCUSSION}

Comme nous en faisions l'hypothèse, nos informateurs évaluent positivement les effets de l'influence lorsqu'il existe une proximité entre les protagonistes de la relation. C'est le cas des situations d'influence décrites comme vécues ou dans les relations d'influence entre amis, dans la famille ou dans l'éducation.

La proximité permet la réciprocité : on peut réagir à l'influence d'une source proche, donner son avis, influencer à son tour... et ainsi, chacun est potentiellement en même temps source et cible de l'influence. Dès lors l'influence n'est pas un mécanisme d’assujettissement, de désindividuation, d’aliénation, mais elle est ce qui dans le rapport à autrui insère et construit l'individu (l'objectif de telles influences est la socialisation, l'éducation, le conseil).

Quand celui qui a le pouvoir menace et impose ses idées, l'influence n'est pas réciproque, pas saine. (L)

L'importance de la réciprocité est saillante dans les domaines du travail et de la politique. Dans ces domaines où les effets de l'influence sont généralement évalués négativement, les influences réciproques sont toujours évaluées positivement. Ainsi, dans le domaine du travail, deux informateurs décrivent les influences d'un syndicat contestataire ou d'un employé engagé et résistant, des influences qui s'opposent aux influences descendantes d'un supérieur ou d'un patron. Dans le domaine de la politique, où les situations d'influence ne sont pas des situations de proximité (aucune situation vécue, grande distance entre source et cible) trois informateurs décrivent positivement les effets d'influences minoritaires opposées à des influences dominantes et contraignantes.

Dans les situations de réciprocité, source et cible existent en tant que sujet l'une pour l'autre. La source influence autrui pour le persuader, le convertir afin qu'il partage ses intérêts, ses opinions, sa vérité (par exemple, les parents qui orientent leur enfant dans la trajectoire qui leur semble bonne, la religion qui dit sa vérité). Par l'influence, la cible mobilise l'intention de la source. L'influence la rend donc plus semblable à la source, et même si elle n'adopte pas le point de vue de la source, elle connaît son intention, ses convictions, 
ses intérêts. Ainsi, les protagonistes de l'influence sont sur le même terrain et participent au même échange ${ }^{13}$. Ceci est évidemment facilité par la proximité.

Par contre dans les situations non réciproques, la source influence autrui pour des raisons qui n’apparaissent pas à la cible (par exemple, des politiciens qui disent ce que les gens veulent entendre pour être élus ou des gourous qui font croire à des balivernes pour s'enrichir). L'intention de la source est inaccessible à la cible et même si la cible change dans le sens de l'influence de la source elle ne se rapprochera pas pour autant de la source dont les intentions sont ailleurs et restent inconnues de la cible. Plus encore, dans les situations de non réciprocité, la cible est niée. En agissant sur autrui sans lui permettre de réagir où en négligeant sa réaction, la source fait une action violente. Et paraphrasant Jacques (1979, p. 55), elle agit comme si elle était seule à agir et que l'univers n'était là que pour recevoir l'impact de son action.

Nos informateurs distinguent ces situations de non réciprocité que Moscovici (1994) qualifie de manipulations. C'est pour cette raison que beaucoup n'évoquent pas la manipulation sectaire et que, lorsque l'interviewer les interroge sur cet aspect, certains commencent par dire que c'est autre chose ou plus que de l'influence.

Si la réciprocité et la proximité de la source d'influence dépendent des caractéristiques des situations décrites, elles dépendent aussi de la manière de situer la source. Or il est possible d'imaginer que derrière une source visible et proche se cache une source lointaine et invisible. Il suffit de supposer cela pour voir des manipulations aux effets néfastes plutôt qu'une relation d'influence réciproque (Laurens, 20I3).

13 C'est cette idée que développe Merleau-Ponty pour ce qui concerne le dialogue: «il se constitue entre autrui et moi un terrain commun, ma pensée et la sienne ne font qu'un seul tissu, mes propos et ceux de l'interlocuteur sont appelés par l'état de la discussion, ils s'insèrent dans une opération commune dont aucun de nous n'est le créateur. II y a là un être à deux, et autrui n'est plus ici pour moi un simple comportement dans mon champ transcendantal, ni d'ailleurs moi dans le sien, nous sommes l'un pour l'autre collaborateurs dans une réciprocité parfaite, nos perspectives glissent l'une dans l'autre, nous coexistons à travers un même monde.» (Merleau-Ponty, 1945, p. 407). 
Ce déplacement imaginaire est parfaitement illustré par un reportage de M6 «Tom Cruise vitrine de la dangereuse secte scientologie». Le reportage montre tout d'abord la source visible: Tom Cruise, son potentiel de séduction, son réseau de relations, etc. Si les qualités de l’acteur sont manifestes, il n’en reste pas moins que rien dans ce que montre ce reportage n'indique comment il influence des gens, les convertit à la scientologie. En outre, si Tom Cruise peut exercer une influence sur autrui, il peut aussi être la cible de l'influence d'autrui. Par exemple, le reportage le montre avec Sarkozy: s'il peut l'influencer, ce dernier peut aussi le faire. Tom Cruise est connu, visible, accessible et il est possible de l'influencer. Dans cette partie du reportage, on ne voit donc pas de manipulation ni de danger.

Ils sont révélés dans la seconde partie du reportage: Tom Cruise n’est pas la source d'influence que nous croyons, mais il est une vitrine, un pantin manipulé par la scientologie. De ce fait, il peut relayer vers nous les influences de la scientologie, mais les influences retour que nous pourrions exercer sur lui n’atteindront jamais la véritable source d'influence. Ce qui pouvait nous apparaître comme une influence réciproque se révèle être une manipulation dès lors que nous supposons que la véritable source est ailleurs et inaccessible. Sur ce point le reportage est particulièrement riche et montre la véritable instance du pouvoir : «l'endroit où la scientologie est contrôlée», «le centre du haut commandement». C'est une «base secrète où la secte élabore sa stratégie mondiale», une base isolée, située en plein désert et même «la plupart des scientologues ignorent l'existence de cet endroit». Cette «forteresse scientologique est placée sous haute protection. Personne ne doit entendre ou voir ce qui se passe derrière ces murs ». Et le reportage montre des images floues prises à grande distance de gros bâtiments gris en détaillant les protections : caméras, gardes, fils barbelés, fusils, etc. Comme on le voit avec ce reportage, il suffit - et c'est toujours possible - de supposer l'existence d'une source inaccessible et invisible (une figure du diable) pour que l'influence devienne une dangereuse manipulation. 


\section{BIBLIOGRAPHIE}

ASSEMBLÉE NATIONALE, 2000, Rapport n²472, enregistré à la Présidence de l'Assemblée nationale le 14 juin: <http://www.assemblee-nationale.fr/l I/ rapports/r2472.asp>.

BERNHEIM Hippolyte, 191/, De la suggestion, Paris, Albin Michel.

CRANO William D., 2012, The Rules of Influence: Winning When You're in the Minority, New York, St Martin's Press.

CROCQ Jean, 1986, L'Hypnotisme scientifique. Rapport à M. le ministre de l'Intérieur et de l'Instruction publique, Paris, Société d'éditions scientifiques.

DAUDET Alphonse, 1888, L'Évangéliste, Paris, Alphonse Lemerre.

DAVISON Walter Phillips, 1983, «The Third-Person Effect in Communication», The Public Opinion Quarterly, 47-I, p. I-I5.

DELBCEUF Joseph, 1993 (I893), «Quelques considérations sur la psychologie de I'hypnotisme, à propos d'un cas de manie homicide guérie par suggestion », in Le Sommeil et les Rêves, Paris, Fayard, p. 405-423.

GABEL Joseph, 1970, Sociologie de l'aliénation, Paris, PUF.

JACQUES Francis, 1979, Dialogiques. Recherches logiques sur le dialogue, Paris, PUF.

JODELET Denise, 2005, «Figures de l'altérité», in L'Autre: Regards psychosociaux, Margarita Sanchez-Mazas et Laurent Licata (éds), Grenoble, PUG, p. 23-47.

JOFFE Hélène, 2005, «"L’Autre" et la construction identitaire: entre dynamiques psychiques et dynamiques sociales », in L'Autre: Regards psychosociaux, Margarita Sanchez-Mazas et Laurent Licata (éds), Grenoble, PUG, p. 95-116.

KATZ Elihu, LAZARSFELD Paul, 2008 (1955), Influence personnelle, Paris, Armand Colin.

KLEIN Melanie, 1946, «Notes on Some Schizoid Mechanisms», International Journal of Psycho-Analysis, 27, p. 99-110.

LAURENS Stéphane, 2005, «La médecine face à l'hypnose. Le congrès de 1889, les prémisses d'un débat actuel? », Revue internationale de psychosociologie, XI-24, p. I55-165.

-, 2013, «Omnipotents manipulateurs invisibles. Comment les influences cachées peuvent tout expliquer», Diogène, 24I-I, p. 102-II4. 
-, MARKOVA Ivana, 20II, «Influence et dialogisme», Bulletin de psychologie, 64-5, p. 387-389.

LAZARSFELD Paul, BERELSON Bernard, GAUDET Hazel, 1944, The People's Choice, New York, Colombia University Press.

LÉVY-BRUHL Lucien, 1949, Carnets, Paris, PUF.

MALEBRANCHE Nicolas, 1946 (1674), De la recherche de la vérité, Paris, Vrin.

MANNHEIM Karl, 1956 (1929), Idéologie et utopie. Une introduction à la sociologie de la connaissance, Paris, Librairie Marcel Rivière et Cie.

MERLEAU-PONTY Maurice, 1945, Phénoménologie de la perception, Paris, Gallimard. MISSIKA Jean-Louis, 1994, «La République des médias», Pouvoirs, 68, p. 101-110.

MOSCOVICI Serge, 1979, Psychologie des minorités actives, Paris, PUF.

-, 1994, «L'influence n'est pas une manipulation», Sciences humaines, 37, 1994, p. 38-41.

-, 2012, Raison et Cultures, Paris, EHESS.

PRISLIN Radmila, CRANO William D., 2012, «A History of Social Influence

Resarch », in Handbook of the history of social psychology, Arie W. Kruglanski et Wolfgang Stroebe (éds), New-York, Psychology Press, p. 321-339.

PROUST Marcel, 1946 [1922], À la recherche du temps perdu, Le Côté de Guermantes, Deuxième partie, Paris, Gallimard.

«Rapport des commissaires chargés par le roi de l'examen du magnétisme animal », I826 (1784), in Alexandre Bertrand, Du magnétisme animal en France et des jugements qu'en ont portés les sociétés savantes. Considérations sur l'apparition de l'extase dans les traitements magnétiques, Paris, J.B. Baillière, p.482-5I0.

ROUSSELLE Aline, 1990, Croire et Guérir. La foi en Gaule dans l'Antiquité tardive, Paris, Fayard.

SANCHEZ-MAZAS Margarita, LICATA Laurent, 2005, «Altérité et changement social», in L'Autre: Regards psychosociaux, Margarita Sanchez-Mazas et Laurent Licata (éds), Grenoble, PUG, p. 337-353.

TURNER John C., 2005, «Explaining the Nature of Power: A Three-Process Theory», European Journal of Social Psychology, 35- I, 2005. p. I-22. 